\title{
Análise da produção científica no Brasil sobre envelhecimento e quedas
}

\author{
Rebeca Fernanda Ferraz de Almeida*, Luciana Araújo Dos Reis**
}

\section{Resumo}

O presente estudo tem por objetivo analisar a produção científica no Brasil sobre envelhecimento e quedas. Trata-se de uma revisão sistemática qualitativa, na qual foram incluídos artigos escritos em português publicados no período de 2011 a 2015. Foram analisadas dezessete publicações que trataram dos temas mais abordados sobre a relação entre quedas e envelhecimento. Mediante a análise de conteúdo de Bardin, emergiram três temas de análise: fatores intrínsecos de risco para quedas, fatores extrínsecos de risco para quedas e intervenção preventiva: contribuições para a saúde dos idosos. Evidenciou-se, ainda, diante da análise do conhecimento produzido por outros autores, que a queda é um fator determinante para o declínio da saúde da população e que há uma interligação frequente entre os fatores de risco tanto intrínsecos como extrínsecos.

Palavras-chave: Envelhecimento. Fatores de riscos. Idosos. Quedas.

\section{Introdução}

A população brasileira, nos últimos anos, passou por uma profunda transformação demográfica. Estudos mostram que o aumento da expectativa de vida da população está relacionado a políticas públicas como: acesso a vacinas, combate à desnutrição, redução das taxas de mortalidade e natalidade (DATASUS, 2015a).

$\mathrm{O}$ envelhecimento traz inúmeras alterações ao indivíduo, no entanto, o estilo de vida também pode influenciar em sua independência. $\mathrm{O}$ processo pode ser natural, quando atrelado às alterações fisiológicas e biológicas, ou patológico, quando acompanhado de doenças. No que tange às alterações naturais, o indivíduo passa a ter restrição física em alguns sistemas do organismo, dentre

* Acadêmica do curso de Fisioterapia da Faculdade Independente do Nordeste. E-mail: rebecafferraza@ gmail.com

** Doutora em Ciências da Saúde pela Universidade Federal do Rio Grande do Norte. Pós-doutora em Saúde Coletiva/ISC pela Universidade Federal da Bahia. Docente da Faculdade Independente do Nordeste. Endereço para correspondência: Av. Luiz Eduardo Magalhães, 1.035. Candeias, Vitória da Conquista, Bahia, CEP 45055-420. E-mail: lucianauesb@yahoo.com.br

$\rightarrow$ http://dx.doi.org/10.5335/rbceh.v13i2.5754

Recebido em: 11/04/2016. Aceito em: 30/08/2016. 
eles, pode-se destacar o sistema musculoesquelético, que apresenta redução da massa muscular e da densidade óssea, enrijecimento dos tendões e ligamentos e aumento da viscosidade do líquido sinovial (FECHINE; TROMPIERI, 2012).

Segundo Andrade et al. (2011), o sistema nervoso central também apresenta mudanças significativas que podem influenciar na resposta motora do idoso. Essas alterações podem influenciar no controle postural dos indivíduos, pois, se não há uma interação completa desses sistemas, torna-se impossível manter o equilíbrio e a postura, levando a uma lentidão da resposta motora e, até mesmo, a episódios de quedas (CARVALHO et al., 2015).

A Organização Mundial de Saúde verificou que 424 mil pessoas morrem por ano devido a quedas. Cerca de $50 \%$ das pessoas com mais de 65 anos sofrem um episódio de quedas ao ano, sendo que a maioria desses idosos que cai apresenta lesões ou sequelas decorrentes da queda. O Instituto Nacional de Traumatologia e Ortopedia realizou uma pesquisa que identificou que $65 \%$ dos pacientes internados haviam adquirido suas lesões ortopédicas provenientes de quedas, indicando aas quedas como um problema de saúde pública (DATASUS, 2015b).

De acordo com dados da Sociedade Brasileira de Geriatria e Gerontologia (2015, p. 3), a queda pode ser definida como: "o deslocamento não intencional do corpo para um nível inferior à posição inicial com incapacidade de correção em tempo hábil, determinado por circunstâncias multifatoriais comprometendo a estabilidade". Na maioria das vezes, ocorre no ambiente domiciliar, contribuindo para o aumento da mortalidade e de morbidades.

Estudos revelam que quando as quedas não causam morte ou lesões, o indivíduo pode desenvolver limitação na qualidade de vida, levando ao receio e à limitação nas atividades de vida diária, estimulando o confinamento e a depressão (ROSA et al., 2015; FECHINE; TROMPIERI, 2012). Estudos mostram que a prevenção é a melhor forma de evitar as quedas, embora, o Brasil ainda necessite de melhorias em seus programas de prevenção, diferente de outros países que dão prioridade a esse fator de risco que influencia diretamente na saúde da população (ROSA et al., 2015; FECHINE; TROMPIERI, 2012).

Em virtude do exposto, evidencia-se que devido ao aumento da população idosa as quedas constituem-se tema de debate para pesquisadores, gestores sociais e políticos. Diversos estudos são desenvolvidos no Brasil sobre quedas e o processo de envelhecimento, buscando discutir os fatores de riscos e as consequências. Nesse contexto, surgiu a questão: como ocorre a produção científica no Brasil sobre envelhecimento e quedas em estudos escritos em português?

Para responder a essa proposição, o presente estudo tem como objetivo analisar a produção científica no Brasil sobre envelhecimento e quedas, permitindo a interação de informações que poderão contribuir para a prevenção. 


\section{Metodologia}

O presente artigo caracteriza-se como uma revisão sistemática qualitativa. A revisão sistemática é um método de pesquisa fundamentado, que investiga um tema sobre o qual o pesquisador produz conhecimento (ERCOLE; MELO; ALCOFORADO, 2014).

Os dados foram obtidos nas bases de dados SciELO e Bireme, utilizando palavras-chaves de acordo os descritores em ciências da saúde (DeCS): envelhecimento e quedas. Para seleção dos dados, foi utilizado o operador booliano AND nas buscas: envelhecimento AND quedas.

Como critérios de inclusão foram adotados: artigos publicados no período de 2011 a 2015, escritos em português e na modalidade de artigo original na íntegra; e como critérios de exclusão, todos os artigos que não informassem sobre o assunto principal e que não apresentassem os descritores adotados neste estudo (envelhecimento AND quedas).

A seleção dos artigos seguiu rigorosamente os itens de inclusão e exclusão. A primeira busca foi realizada na base de dados SciELO, que processou 58 artigos, dentre esses, 25 foram eliminados por não atender aos critérios de inclusão, sendo assim, 33 artigos foram selecionados. Ao realizar a busca na base de dados, Bireme, foram processados 1.100 artigos, dos quais, 1.020 foram eliminados por não estarem dentro dos critérios de inclusão, sendo assim, 80 foram selecionados.
Para os artigos selecionados nas duas bases de dados (113 artigos), foi realizada leitura do título e do resumo. Assim, 64 artigos foram excluídos por não apresentarem título de acordo com objetivo do estudo e 32 foram eliminados após leitura completa dos artigos, portanto, ao final, foram selecionados 17 artigos para o estudo, conforme sistematizado na Figura 1.

Figura 1 - Fluxograma de seleção dos estudos de acordo com os critérios de inclusão e exclusão a partir dos descritores: envelhecimento AND quedas

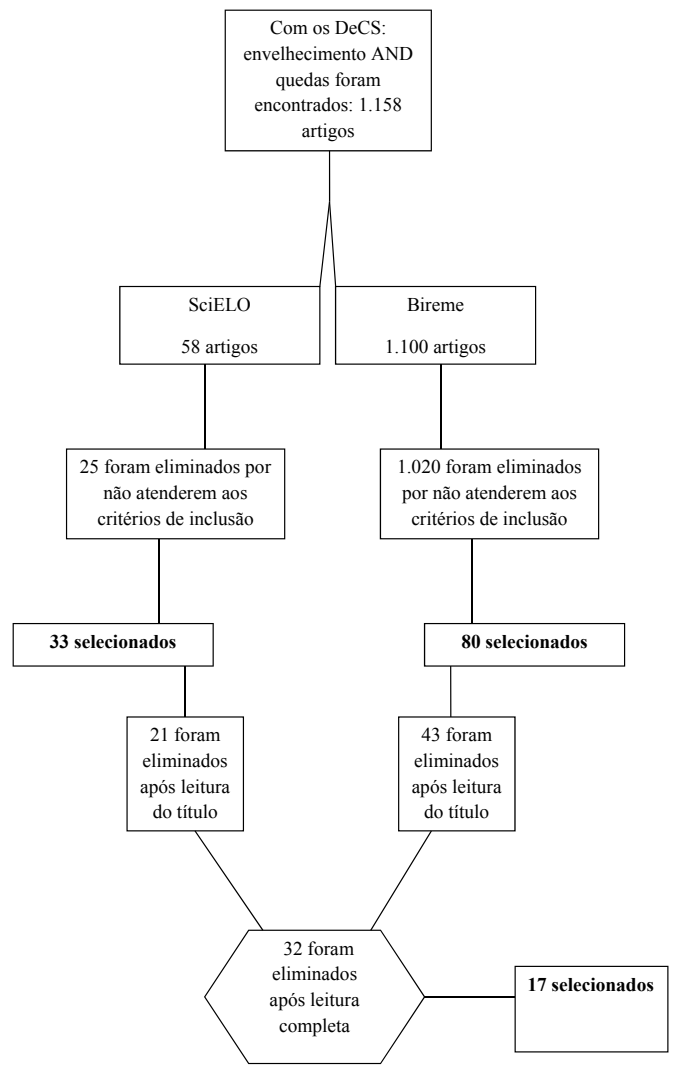

Fonte: dados da pesquisa. 
As informações coletadas nos artigos foram analisadas utilizando-se a técnica de análise temática fundamentada na análise de conteúdo de Bardin (2010). Porém, devido à grande quantidade de informações obtidas nos artigos, tornou-se necessário o uso de uma ferramenta computacional de suporte para análise dos dados e para auxiliar no manuseio e na organização dos dados, facilitando o processo de integração entre as categorias emergentes. Para tanto, utilizou-se o software de tratamento de dados qualitativos QSR NVivo ${ }^{\circledR}$, versão 11.0, doravante escrito como NVivo.

O processo de análise de conteúdo foi operacionalizado em três etapas: pré-análise, exploração do material e tratamento dos resultados: inferência e interpretação (BARDIN, 2010). Na etapa de pré-análise, os artigos foram inseridos no NVivo com o recurso de importação de fontes de informação, compondo, assim, o corpus da pesquisa. Após a criação do banco de dados no NVivo, deu-se início à etapa seguinte, a de exploração do material, quando foi feita a leitura minuciosa dos artigos e realizado o processo de codificação com a decomposição dos conteúdos em unidades de registro com base nas expressões com sentidos equivalentes que surgiram ao longo do corpus da pesquisa, que foram agrupadas nas categorias analíticas emergentes dos dados empíricos.

Nessa etapa, utilizou-se a técnica nuvem de palavras, do Nvivo, para análise do material empírico. Essa técnica pode ser compreendida como uma forma de visualização de dados linguísticos, que mostra a frequência com que as palavras aparecem em um dado contexto. A técnica de construção dessa nuvem consiste em usar tamanhos e fontes de letras diferentes de acordo com as ocorrências das palavras na categoria analisada, gerando uma imagem que apresenta um conjunto de palavras, coletadas do corpo do texto e agregadas de acordo com sua frequência, sendo que as palavras mais frequentes aparecem no centro da imagem e as demais em seu entorno, de modo decrescente. Dessa maneira, essa técnica contribui para a visualização do que é mais relevante nos artigos selecionados.

De posse da nuvem de palavras e dos dados codificados, foi iniciada a terceira e última etapa, a do tratamento dos resultados. Buscou-se a articulação entre o material empírico e o referencial teórico, o que permitiu a ocorrência de outras contribuições teóricas sugeridas pela leitura do material empírico.

\section{Resultados e discussão}

A caracterização dos artigos analisados, conforme autor, tipo de estudo, amostra, local, periódico e data de publicação, encontra-se descrita na Tabela 1. 
Tabela 1 - Caracterização da produção científica selecionada

\begin{tabular}{|c|c|c|c|c|c|}
\hline $\begin{array}{c}\text { Autor/ } \\
\text { referência }\end{array}$ & Tipo de estudo & Amostra & Local & Periódico & Ano \\
\hline $\begin{array}{l}\text { Alves; } \\
\text { Scheicher }\end{array}$ & Transversal & 88 idosos & $\begin{array}{l}\text { Lar dos Velhos Frederico Oza- } \\
\text { nan e Lar São Vicente de Pau- } \\
\text { lo, da cidade de Garça, São } \\
\text { Paulo }\end{array}$ & $\begin{array}{l}\text { Revista Brasileira } \\
\text { de Geriatria e Ge- } \\
\text { rontologia }\end{array}$ & 2011 \\
\hline Alveiro et al. & Transversal & 739 idosos & $\begin{array}{l}\text { Área de abrangência da Estra- } \\
\text { tégia Saúde da Família do mu- } \\
\text { nicípio de São Carlos }\end{array}$ & $\begin{array}{l}\text { Ciência \& Saúde } \\
\text { Coletiva }\end{array}$ & 2012 \\
\hline $\begin{array}{l}\text { Battistella; } \\
\text { Alfieri }\end{array}$ & Transversal & 26 idosos & $\begin{array}{l}\text { Hospital Universitário Mútua de } \\
\text { Terrassa, Barcelona, Espanha }\end{array}$ & Acta Fisiátrica & 2011 \\
\hline $\begin{array}{l}\text { Burgos; } \\
\text { Carvalho }\end{array}$ & $\begin{array}{l}\text { Descritivo, comparati- } \\
\text { vo, de corte transversal }\end{array}$ & 75 idosos & $\begin{array}{l}\text { Laboratório do Sono da Clínica } \\
\text { de Pneumologia do Hospital } \\
\text { das Forças Armadas de Bra- } \\
\text { sília, DF }\end{array}$ & $\begin{array}{l}\text { Fisioterapia em } \\
\text { Movimento }\end{array}$ & 2012 \\
\hline $\begin{array}{l}\text { Campos; } \\
\text { Vianna; Campos }\end{array}$ & $\begin{array}{l}\text { Prospectivo, transver- } \\
\text { sal e observacional }\end{array}$ & 155 idosos & Hospital Público de Brasília & $\begin{array}{l}\text { Revista Kairós } \\
\text { Gerontologia }\end{array}$ & 2013 \\
\hline $\begin{array}{l}\text { Carvalho; } \\
\text { Luckow; } \\
\text { Siqueira }\end{array}$ & $\begin{array}{l}\text { Delineamento trans- } \\
\text { versal }\end{array}$ & 195 idosos & $\begin{array}{l}19 \text { instituições, casas para } \\
\text { idosos, asilos e clínicas geriá- } \\
\text { tricas do município de Pelotas }\end{array}$ & $\begin{array}{l}\text { Ciência \& Saúde } \\
\text { Coletiva }\end{array}$ & 2011 \\
\hline $\begin{array}{l}\text { Cavalcante; } \\
\text { Aguiar; Gurgel }\end{array}$ & $\begin{array}{l}\text { Transversal, descritivo } \\
\text { com abordagem quan- } \\
\text { titativa }\end{array}$ & 50 idosos & $\begin{array}{l}\text { Bairro Cidade 2000, de Forta- } \\
\text { leza }\end{array}$ & $\begin{array}{l}\text { Revista Brasileira } \\
\text { de Geriatria e } \\
\text { Gerontologia } \\
\end{array}$ & 2012 \\
\hline Cruz et al. & Transversal & 213 idosos & $\begin{array}{l}\text { Departamento de Epidemio- } \\
\text { logia da Faculdade de Saúde } \\
\text { Pública }\end{array}$ & Revista Dor & 2011 \\
\hline Cruz et al. & Transversal & 462 idosos & $\begin{array}{l}\text { Residentes na Zona Norte da } \\
\text { cidade de Juiz de Fora, Minas } \\
\text { Gerais }\end{array}$ & $\begin{array}{l}\text { Caderno de Saú- } \\
\text { de Coletiva }\end{array}$ & 2015 \\
\hline $\begin{array}{l}\text { Gasparotto; } \\
\text { Falsarella; } \\
\text { Coimbra }\end{array}$ & Revisão de literatura & - & 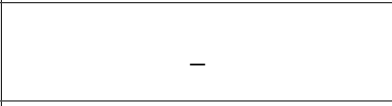 & $\begin{array}{l}\text { Revista Brasileira } \\
\text { de Geriatria e Ge- } \\
\text { rontologia }\end{array}$ & 2014 \\
\hline Pereira et al. & Descritivo e transversal & 6.751 idosos & $\begin{array}{l}\text { Domicílios de } 59 \text { cidades do } \\
\text { estado do Rio Grande do Sul }\end{array}$ & $\begin{array}{l}\text { Ciência \& Saúde } \\
\text { Coletiva }\end{array}$ & 2013 \\
\hline Pinho et al. & $\begin{array}{l}\text { Epidemiológico de cor- } \\
\text { te transversal com abor- } \\
\text { dagem quantitativa }\end{array}$ & 150 idosos & $\begin{array}{l}\text { Unidade de Saúde da Família } \\
\text { Viver Bem }\end{array}$ & $\begin{array}{l}\text { Revista da Esco- } \\
\text { la de Enferma- } \\
\text { gem da USP }\end{array}$ & 2012 \\
\hline Prata et al. & Seccional & 78 idosos & $\begin{array}{l}\text { Projeto Prev-Quedas (Univer- } \\
\text { sidade Federal Fluminense) }\end{array}$ & $\begin{array}{l}\text { Fisioterapia em } \\
\text { Movimento }\end{array}$ & 2011 \\
\hline Prata et al. & $\begin{array}{l}\text { Pesquisa de campo } \\
\text { qualitativa }\end{array}$ & 12 idosos & $\begin{array}{l}\text { Universidade Federal Flumi- } \\
\text { nense com participantes do } \\
\text { projeto Prevenção de Quedas }\end{array}$ & $\begin{array}{l}\text { Revista de Pesqui- } \\
\text { sa Cuidado é Fun- } \\
\text { damental Online }\end{array}$ & 2014 \\
\hline $\begin{array}{l}\text { Rezende; } \\
\text { Carrilho; } \\
\text { Sebastião }\end{array}$ & Revisão sistemática & - & (- & $\begin{array}{l}\text { Caderno de Saú- } \\
\text { de Pública }\end{array}$ & 2012 \\
\hline $\begin{array}{l}\text { Rodrigues; } \\
\text { Fraga; Barros }\end{array}$ & Transversal & 1.520 idosos & $\begin{array}{l}\text { Residentes em área urbana do } \\
\text { município de Campinas, São } \\
\text { Paulo }\end{array}$ & $\begin{array}{l}\text { Revista Brasileira } \\
\text { de Epidemiologia }\end{array}$ & 2014 \\
\hline Silva et al. & $\begin{array}{l}\text { Observacional, descri- } \\
\text { tivo e transversal }\end{array}$ & 47 idosos & $\begin{array}{l}\text { Três instituições de longa perma- } \\
\text { nência de Teresina, Pl: Fundação } \\
\text { Abrigo São Lucas, Vila do Ancião } \\
\text { e Casa Frederico Ozanam }\end{array}$ & $\begin{array}{l}\text { Revista Brasileira } \\
\text { de Geriatria e } \\
\text { Gerontologia }\end{array}$ & 2013 \\
\hline
\end{tabular}

Fonte: dados da pesquisa. 
Conforme a Figura 2, as palavras mais frequentes nos artigos avaliados, em ordem decrescente, foram: "quedas", "idosos", "risco" e "saúde". Com base nessas palavras, três temas de análise emergiram: fatores intrínsecos de risco para quedas; fatores extrínsecos de risco para quedas; intervenção preventiva: contribuições para a saúde dos idosos.

Figura 2-Nuvem de palavras elaborada com base nos artigos selecionados para análise

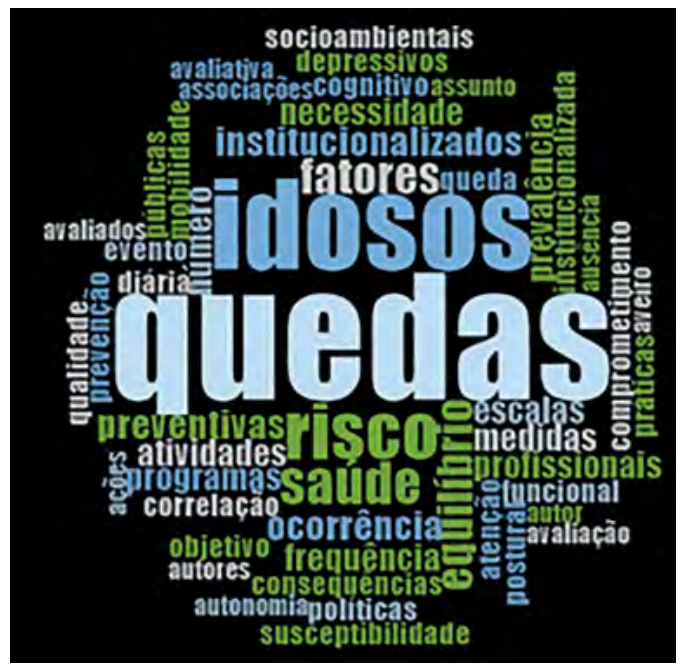

Fonte: dados da pesquisa.

A queda durante o processo do envelhecimento é associada a outros fatores, sejam eles intrínsecos ou extrínsecos. Esse fato levou os autores selecionados a identificar e detalhar quais são os riscos mais frequentes envolvidos nesse fenômeno. Segundo Rodrigues, Fraga e Barros (2014) os intrínsecos são os fatores decorrentes de alterações fisiológicas relacionadas ao envelhecimento, às doenças e aos efeitos de fármacos, e extrínsecos são fatores relativos às circunstâncias e condições ambientais.

\section{Fatores intrínsecos de risco para quedas}

Campos, Vianna e Campos (2013) avaliaram os fatores de risco para quedas por meio do teste de equilíbrio, do teste de alcance funcional e do Timed Up \& Go, e identificam aqueles com maiores chances de cair. Os autores identificaram como risco três variáveis importantes: sexo, polifarmácia e medo de cair, dentre esses, a polifarmácia foi a mais significativa estatisticamente. Além disso, ao avaliar os testes de equilíbrio, puderam identificar que não houve relação significativa com o risco de quedas. Concluíram que o fator de risco para quedas mais evidente foi o uso de polifarmácia, explicando que o uso de medicamentos pode "ocasionar hipotensão postural e alterações no sistema nervoso central, levando a distúrbios na visão, na propriocepção, no equilíbrio e na coordenação, com consequências mais acentuadas quando há uso concomitante de várias drogas" (CAMPOS; VIANNA; CAMPOS, 2013, p. 133).

Um estudo realizado por Burgos e Carvalho (2012) teve como objetivo avaliar a relação entre a síndrome da apneia obstrutiva do sono (Saos) e a sonolência diurna excessiva com os riscos e eventos de quedas em idosos. Foi possível identificar que dos três grupos avaliados, todos apresentavam a sonolência diurna e quanto maior a severidade da Saos maior o grau de sonolência diurna. Os autores explicam que 
[...] a sonolência diurna excessiva se torna de grande relevância, visto que leva a prejuízo de funções cognitivas, tais como concentração, atenção e memória (BURGOS; CARVALHO, 2012, p. 101).

Apesar desses achados, o estudo não obteve resultados estatisticamente significativos referentes à correlação entre os riscos de quedas.

Outro fator de risco está relacionado a doenças crônicas que incluem como sintoma principal a dor. Para Cruz et al., "[...] a prevalência de queda e dor crônica é elevada em idosos com importantes consequências para a qualidade de vida" (2011, p. 108). Eles realizaram um estudo com 213 idosos com dor crônica há um ano e com episódios de quedas com o objetivo de verificar a prevalência das quedas e associar o local, a intensidade e o tempo da dor. Obtiveram como resultado a prevalência de $56 \%$ de maior risco de quedas para os idosos que sofreram a ocorrência de quedas e os idosos com dor moderada a intensa do que os com dor leve. Alguns estudos comprovam que a dor é um fator determinante para a redução do balanço e da mobilidade em idosos.

Segundo Cruz et al.,

[...] o idoso com comprometimento cognitivo pode apresentar déficits de mobilidade, lentificação de movimentos, alterações comportamentais e menor tempo de reação frente aos desequilíbrios (2015, p. 390).

Sendo assim, essas alterações tornam-se preditores para quedas. A capacidade cognitiva tem significativa relação com ocorrências de quedas, isso ocorre porque os sistemas que desenvolvem a função motora agem com uma interligação com o sistema cognitivo e para a realização de uma tarefa é necessário ter uma atenção que irá influenciar nos comandos motores até a execução da tarefa.

A depressão é uma das doenças que acometem a população idosa, existem estudos que revelam a relação entre depressão e quedas. Um estudo com 78 idosos participantes de um projeto de prevenção a quedas identificou que $21,8 \%$ tinham depressão e $32 \%$ haviam caído no último ano. Os autores explicam que o uso de medicamentos antidepressivos pode causar efeitos colaterais e pode ocasionar sintomas relativos ao quadro depressivo, como "indiferença ao meio ambiente, adulteração do nível da atenção, redução do comprimento da passada, diminuição da energia, diminuição da autoconfiança, reclusão, inatividade e perdas cognitivas" (PRATA et al., 2011, p. 441). Dessa forma, fica evidente que a depressão pode influenciar na ocorrência de quedas.

Segundo Alveiro et al. (2012), a mobilidade dos idosos pode influenciar em episódios de quedas, pois eles passam a ter mais dificuldades físicas para executar as tarefas da vida diária. Os autores revelaram que os idosos avaliados por meio do teste Timed Up \& Go apresentaram menor mobilidade e maior risco de quedas, explicando que o processo de envelhecimento leva a reduções de massa, força e qualidade do músculo, além das alterações do equilíbrio, dessa forma, influenciam na mobilidade do indivíduo.

Silva et al. (2013), em estudo realizado com idosos institucionalizados, identi- 
ficaram que a maioria dos idosos obteve baixa pontuação na escala de equilíbrio e a maior dependência foi entre as mulheres. Os autores explicam que o risco de quedas pode estar relacionado à redução da realização das atividades de vida diária e à falta de equilíbrio, além disso, enfatizam que a maioria dos idosos institucionalizados é sedentária, o que aumenta o declínio funcional e o risco de quedas.

Diante dos dados apresentados, observou-se que o principal fator intrínseco para o risco de quedas é o comprometimento do equilíbrio, sendo assim, são necessárias medidas de reabilitação que visem melhorar o equilíbrio e o comprometimento funcional dos idosos, de forma a reduzir as quedas e, consequentemente, melhorar a qualidade de vida desses sujeitos.

\section{Fatores extrínsecos de risco para quedas}

Pinho et al. (2012) realizaram um estudo com 150 idosos de uma comunidade, revelando que as principais causas de quedas estão relacionadas a fatores de origem extrínseca. Os autores explicam que a maioria dos idosos passa a maior parte do tempo em casa e, geralmente, não há uma adequação do ambiente, proporcionando um maior risco de quedas.

O estudo realizado por Pereira et al. (2013) teve como objetivo avaliar os fatores socioambientais quando relacionados a quedas. Os autores utilizaram como amostra uma população de 6.715 idosos de 59 cidades. Os autores identificaram que para os idosos que consideram o ambiente urbano inseguro esse acaba sendo um fator de proteção, pois eles evitam ou circulam com mais cuidado, embora, acabem se socializando menos. Já aqueles que têm boa percepção do ambiente urbano podem estar mais expostos a riscos de quedas, pois acabam sendo menos cuidadosos. Os autores concluíram que há a necessidade de uma readaptação do ambiente urbano para minimizar os riscos de quedas e torná-lo mais colaborativo para atividades comunitárias.

Prata et al. (2014) realizaram uma pesquisa qualitativa na qual observaram que as quedas têm causas multifatoriais, não somente por conta da idade ou do declínio das funções, mas também por haver uma grande parcela de "culpa" relacionada a fatores de má conservação dos ambientes públicos, como iluminação, calçadas, transportes públicos, sinalização, entre outros fatores que podem provocar quedas.

Carvalho, Luckow e Siqueira (2011) identificaram maior prevalência de quedas nos idosos institucionalizados, sendo o local mais frequente em frente ao quarto. Eles explicam que havia um número excessivo de idosos nos quartos e que não havia espaço livre para circulação, o que aumenta os riscos de quedas se não houver a intervenção de medidas que possam adequar o ambiente.

Sabendo que os fatores intrínsecos são de origem fisiológica, Rezende, Carrillo e Sebastião (2012) pesquisaram a respeito da relação entre uso de medicamento e quedas. Os autores 
identificaram que o uso de diuréticos e psicoativos em geral representa um potencial fator de risco de quedas. No caso dos diuréticos, eles explicam que podem gerar como efeitos colaterais hipotensão postural, vertigem e fraqueza, além disso, os psicoativos também podem causar hipotensão, arritmias, sedação, tremores e relaxamento muscular.

Com base nas informações apresentadas, evidencia-se que com a adoção de medidas preventivas o risco de quedas pode ser reduzido, pois o simples planejamento do ambiente de residência do idoso já reduz consideravelmente o risco de quedas. Desse modo, é importante maior divulgação e a adoção das medidas preventivas contra quedas, de forma a sensibilizar a sociedade, em especial os próprios idosos, sobre a necessidade da adoção de tais medidas.

Intervenção preventiva: contribuições para a saúde dos idosos

As quedas de idosos são um problema de saúde pública, pois podem interferir diretamente na saúde dessa população. Nesse sentido, Battistella e Alfieri (2011) avaliaram um programa de prevenção do Hospital Universitário Mútua de Terrassa, Barcelona, na Espanha, que trabalha com idosos que sofreram quedas ou têm risco eminente de sofrer quedas, tendo como objetivo avaliar os indivíduos por uma equipe multidisciplinar (médico geriatra, médico fisiatra, fisioterapeuta e enfermeira), educar os idosos por meio de vídeos e palestras, visando mostrar os riscos mais frequentes que podem levar a quedas. Além disso, esse programa promove atividades físicas que proporcionam alongamento muscular, fortalecimento muscular, estimulação do equilíbrio, coordenação e propriocepção. Os autores concluíram que o serviço é muito importante para a prevenção de quedas e deve servir como modelo.

Para Silva et al. (2013), idosos institucionalizados tornam-se menos ativos, dessa forma, é necessário que os responsáveis tenham uma visão mais focada em cada idoso, pois cada um tem uma necessidade individual. Além disso, os autores enfatizam a importância de manter o idoso ativo, utilizando atividades em grupo ou individuais.

Um estudo realizado por Alves e Scheicher (2011) identificou que o idoso institucionalizado tem nove vezes mais chances de cair do que aquele que não é institucionalizado. No entanto, o idoso pode ter seu equilíbrio treinado e estimulado por meio das atividades físicas que são realizadas durante o dia, o que não acontece com muitos idosos institucionalizados, acarretando no déficit em equilíbrio e aumentando o risco de quedas. Os autores salientam que devem ser praticados exercícios relacionados com velocidade, amplitudes variadas, mudanças de direção e em locais diferentes.

Quando se fala sobre a importância de um planejamento adequado nas construções, pensando na diversidade de pessoas que nelas circulam, os idosos representam a maior preocupação. Prata et al. afirmam que deve haver: 
[...] adequação dos espaços públicos de lazer e circulação, além de adaptações nos espaços urbanos para permitir a participação segura do idoso na vida em sociedade, redução da altura do meio fio das calçadas, melhoria do calçamento $(2014$, p. 692$)$.

Dessa forma, verifica-se que há a necessidade de que as práticas de prevenção contra quedas ocorram de forma mais efetiva e eficaz, a fim de evitar as possíveis consequências e sequelas decorrentes. Ressalta-se que as quedas representam um elevado custo para o sistema de saúde e é um importante marcador de declínio funcional e comprometimento da qualidade de vida.

\section{Conclusão}

Constatou-se nos estudos analisados que os temas mais abordados nos artigos foram: fatores intrínsecos de risco para quedas; fatores extrínsecos de risco para quedas; e intervenção preventiva: contribuições para a saúde dos idosos. Evidenciou-se, ainda, diante da análise do conhecimento produzido por pesquisadores, que quedas é um fator determinante para o declínio da saúde da população e que há uma interligação frequente entre os fatores de risco tanto intrínsecos como extrínsecos, pois, na maioria dos estudos, fica evidente a relação entre os fatores relacionados ao envelhecimento e os fatores ambientais.

Dessa forma, é necessário que políticas públicas de saúde sejam laboradas no intuito de promover a adoção de medidas preventivas contra quedas, de forma a contribuir com o envelhecimento ativo.

\section{Analysis of scientific production in Brazil on aging and falls}

\section{Abstract}

This study aims to analyze the scientific production in Brazil on aging and falls. This is a qualitative systematic review were included articles published between 2011-2015 written in Portuguese. 17 were analyzed as to which the most discussed topics on the relationship between falls and aging. By Bardin content analysis emerged three analytical themes: intrinsic risk factors for falls; Extrinsic risk factors for falls; and preventive intervention: contributions to the health of the elderly. It was evident even before the analysis of the knowledge produced by others, the falls are a determining factor for the declining health of the population and there is a common link between the both intrinsic and extrinsic risk factors.

Keywords: Aging. Elderly. Falls. Risk factors.

\section{Referências}

ALVEIRO, M. C. et al. Mobilidade e risco de quedas de população idosa da comunidade de São Carlos. Ciência \& Saúde Coletiva, São Paulo (SP), v. 17, n. 9, p. 2481-2488, 2012.

ALVES, N. B.; SCHEICHER, M. E. Equilíbrio postural e risco para queda em idosos da cidade de Garça, SP. Revista Brasileira de Geriatria e Gerontologia, Rio de Janeiro (RJ), v. 14, n. 4, p. 763-768, 2012.

ANDRADE, L. P. et al. Efeitos de tarefas cognitivas no controle postural de idosos: uma revisão sistemática. Revista Motricidade, Ribeira de Pena (PO), v. 7, n. 3, p. 19-28, 2011.

BARDIN, L. Análise de Conteúdo. 5 ed. Lisboa: Edições 70, 2010. 
BATTISTELLA, L. R.; ALFIERI, F. M. Unidade de quedas: uma possibilidade de prevenção de quedas em idosos. Acta Fisiátrica, São Paulo (SP), v. 18, n. 1, p. 45-48, 2011.

BURGOS, A. F.; CARVALHO, G. A. Síndrome da apneia obstrutiva do sono (Saos) e sonolência diurna excessiva (SDE): influência sobre os riscos e eventos de queda em idosos. Fisioterapia em Movimento, Curitiba (PR), v. 25, n. 1, p. 93-103, 2012.

CAMPOS, M. P. S.; VIANNA, L. G.; CAMPOS, A. R. Os testes de equilíbrio Alcance Funcional e "Timed Up and Go" e o risco de quedas em idosos. Revista Kairós Gerontologia, São Paulo (SP), v. 16, n. 4, p. 125-138, 2013.

CARVALHO, I. F. et al. Uso da bandagem infrapatelar no desempenho físico e mobilidade funcional de idosas com história de quedas. Revista Brasileira de Geriatria e Gerontologia, Rio de Janeiro (RJ), v. 18, n. 1, p. 119-127, 2015.

CAVALCANTE, A. L. P.; AGUIAR, J. B.; GURGEL, L. A. Fatores associados a quedas em idosos residentes em um bairro de Fortaleza, Ceará. Revista Brasileira de Geriatria e Gerontologia, Rio de Janeiro (RJ), v. 15, n. 1, p. 137-146, 2012.

CARVALHO, M. P.; LUCKOW, E. L. T.; SIQUEIRA, F. V. Quedas e fatores associados em idosos institucionalizados no município de Pelotas (RS, Brasil). Ciência \& Saúde Coletiva, Rio de Janeiro (RJ), v. 16, n. 6, p. 2945-2952, 2011.

CRUZ, D. T. et al. Associação entre capacidade cognitiva e ocorrência de quedas em idosos. Caderno de Saúde Coletiva, Rio de Janeiro (RJ), v. 23, n. 4, p. 386-393, 2015.

CRUZ, H. M. F. et al. Quedas em idosos com dor crônica: prevalência e fatores associados. Revista Dor, São Paulo, v. 12, n. 2, p. 108-114, 2011.

DATASUS. Portal da Saúde. 2015a. Disponível em: <http://portalsaude.saude.gov. br/index.php/cidadao/principal/agencia-saude/19290-pesquisa-traz-retrato-inedito- -da-saude-do-idoso-no-brasil>. Acesso em: 1 mar. 2016

DATASUS. Portal da Saúde. 2015b. Disponível em: <http://portalsaude.saude.gov. br/index.php/cidadao/principal/agencia-saude/18098-campanha-pretende-reduzir-fraturas-por-queda>. Acesso em: 9 mar. 2016 ERCOLE, F. F.; MELO, L. S.; ALCOFORADO, C. L. G. Revisão integrativa versus revisão sistemática. Revista Mineira de Enfermagem, Belo Horizonte (MG), v. 18, n. 1, p. 1-260, 2014.

FECHINE, B. R. A.; TROMPIERI, N. O processo de envelhecimento: as principais alterações que acontecem com o idoso com o passar dos anos. Revista Científica Internacional, Rio de Janeiro (RJ), v. 1, n. 7, p. 106-194, 2012.

GASPAROTTO, L. P. R.; FALSARELLA, G. R.; COIMBRA, A. M. V. As quedas no cenário da velhice: conceitos básicos e atualidades da pesquisa em saúde. Revista Brasileira de Geriatria e Gerontologia, Rio de Janeiro (RJ), v. 17, n. 1, p. 201-209, 2014.

PEREIRA, G. N. et al. Fatores socioambientais associados à ocorrência de quedas em idosos. Ciência \& Saúde Coletiva, Rio de Janeiro (RJ), v. 18, n. 12, p. 3.507-3.514, 2013.

PINHO, T. A. M. et al. Avaliação do risco de quedas em idosos atendidos em Unidade Básica de Saúde. Revista da Escola de Enfermagem USP, São Paulo (SP), v. 46, n. 2, p. 320-327, 2012.

PRATA, H. L. et al. Relatos de quedas extrínsecas em idosos participantes do projeto prev-quedas. Revista de Pesquisa Cuidado é Fundamental Online, São Paulo (SP), v. 6, n. 2, p. 685-694, 2014.

PRATA, H. L. et al. Envelhecimento, depressão e quedas: um estudo com os participantes do Projeto Prev-Quedas. Fisioterapia em Movimento, Curitiba (PR), v. 24, n. 3, p. 437-443, 2011.

REZENDE, C. P.; CARRILHO, M. R. G. G.; SEBASTIÃO, E. C. O. Queda entre idosos 
no Brasil e sua relação com o uso de medicamentos: revisão sistemática. Cadernos de Saúde Pública, Rio de Janeiro (RJ), v. 28, n. 12, p. 2223-2235, 2012.

ROSA, T. S. M. et al. Perfil epidemiológico de idosos que foram a óbito por queda no Rio Grande do Sul. Revista Brasileira de Geriatria e Gerontologia, Rio de Janeiro (RJ), v. 18, n. 1, p. 59-69, 2015.

RODRIGUES, I. G.; FRAGA, G. P.; BARROS, M. B. A. Quedas em idosos: fatores associados em estudo de base populacional. Revista Brasileira de Epidemiologia, Rio de Janeiro (RJ), p. 705-718, jul./set. 2014.

SILVA, J. M. N. et al. Correlação entre o risco de queda e autonomia funcional em idosos institucionalizados. Revista Brasileira de Geriatria e Gerontologia, Rio de Janeiro (RJ), v. 16, n. 2, p. 337-346, 2013.

SOCIEDADE BRASILEIRA DE GERIATRIA E GERONTOLOGIA. Projeto Diretrizes Quedas em idosos: prevenção. 2015. p. 1-10. 